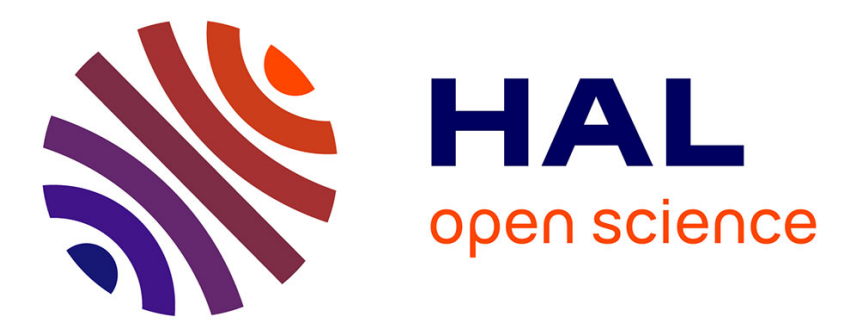

\title{
Entropy complexity analysis of electroencephalographic signals during pre-ictal, seizure and post-ictal brain events
}

\author{
Amira J. Zaylaa, A Harb, Faten I. Khatib, Z Nahas, Fadi N. Karameh
}

\section{To cite this version:}

Amira J. Zaylaa, A Harb, Faten I. Khatib, Z Nahas, Fadi N. Karameh. Entropy complexity analysis of electroencephalographic signals during pre-ictal, seizure and post-ictal brain events. 2015 International Conference on Advances in Biomedical Engineering (ICABME), Sep 2015, Beirut, Lebanon. 10.1109/ICABME.2015.7323270 . inserm-01238586

\section{HAL Id: inserm-01238586 https://www.hal.inserm.fr/inserm-01238586}

Submitted on 18 Dec 2015

HAL is a multi-disciplinary open access archive for the deposit and dissemination of scientific research documents, whether they are published or not. The documents may come from teaching and research institutions in France or abroad, or from public or private research centers.
L'archive ouverte pluridisciplinaire HAL, est destinée au dépôt et à la diffusion de documents scientifiques de niveau recherche, publiés ou non, émanant des établissements d'enseignement et de recherche français ou étrangers, des laboratoires publics ou privés. 


\title{
Entropy Complexity Analysis of Electroencephalographic Signals During Pre-Ictal, Seizure and Post-Ictal Brain Events
}

\author{
A. J. Zaylaa ${ }^{1}$, A. Harb ${ }^{2}$, F. I. Khatib ${ }^{3}$, Z. Nahas ${ }^{4}$ and F. N. Karameh ${ }^{1}$ \\ ${ }^{1}$ Department of Electrical and Computer Engineering, American University of Beirut, Lebanon \\ az56@aub.edu.lb and fk14@aub.edu.lb \\ ${ }^{2}$ Faculty of Engineering, Lebanese University, Lebanon. harb_ahmad@hotmail.com \\ ${ }^{3}$ Sharp Chula Vista Medical Center, California, U.S.A. Faten.khatib@sharp.com \\ ${ }^{4}$ Department of Psychiatry, American University of Beirut, Lebanon. zn17@aub.edu.lb
}

\begin{abstract}
Epileptic seizures reflect runaway excitation that severely hinders normal brain functions. With EEG recordings reflecting real-time brain activity, it is essential to both predict seizures and improve the classification of seizures in EEG signs. Towards this aim, nonlinear tools are strongly recommended to select the seizure-sensitive features prior to classification. However, the choice of the feature remains challenging. With the multitude of entropy parameters available in literature, and in order to perform a judicious selection of features that are fed to classifiers, this paper presents a comparative study of a host of candidate promising feature extraction techniques. Four entropy features namely Approximate Entropy, Sample Entropy and Renyi entropy of order 2 and Renyi entropy of order 3 , were implemented as the standard techniques. Three kernelbased features namely Triangular Entropy, Spherical Entropy and Cauchy entropy were implemented. The former and latter entropies were computed from EEG recordings during induced seizures in three distinct phases: the pre-ictal (pre-seizure) phase, the ictal (seizure) phase, and the post-ictal (post-seizure) phase. Results showed that, among kernel-based methods, Spherical entropy features exhibited the largest parameter sensitivity to (Seizure-Normal) phase changes with the highest normalized relative separation $(100 \%)$. The sample entropy feature in turn showed the most sensitive to EEG phase changes with the highest relative separation $(94.85 \%)$, among the studied entropy alternatives.
\end{abstract}

Index Terms-Approximate Entropy, Sample Entropy, Renyi Entropy, Kernel-based Entropy, EEG, Seizure, Detection.

\section{INTRODUCTION}

Normal brain operation is contingent on the orchestration of neural firing activity among a neuronal subtypes over several scales $\left(10^{-6}-10^{-2} \mathrm{~m}\right)$. Principally, the endogenous balance of excitation and inhibition (positive and negative drives), allows for information transfer, storage and retrieval. Epileptic activity is a transient symptom of excessive or synchronous neuronal activity in the brain [1] that arises either spontaneously (in pathology) or due to external stimulation (in therapeutic protocols). The Epilepsy is mainly assessed using EEG recordings obtained from multiple electrodes placed on the scalp (or over the cortical surface pre-operatively). Subsequent analysis of collected data aim to locate epileptic focus and propagation patterns over brain area [2], [3], a time-consuming task that is often conducted by experienced physicians. Therefore, the development of automated signal analysis techniques that aid in identifying the occurrence of an ictal (epileptic) event, as well as the eminence of such event, continue to be a highly valuable endeavor.

Towards the general aim of distinguishing epileptic from normal EEG signals, several studies have embarked on using nonlinear methods to detect and quantify characteristics changes in nonlinear EEG dynamics. Babloyantz et al. [4] used nonlinear parameters like Correlation Dimension (CD) and Largest Lyapunov Exponents (LLE) to study the sleep wave signal. Non-linear dynamical methods that are based on the chaos theory have been applied to biological signals [5], with epilepsy identification being one of the most common areas. In 2001, Andrzejak et al. [6] presented a framework that used a new measure designed to discriminate between nonlinear deterministic and linear stochastic dynamics of EEG signals. Their results demonstrated that signals from within the epileptogenic zone had strong indications of nonlinear determinism, while those from other sites had linear stochastic dynamics. This was a particular indication that nonlinear time series analysis of EEG signals might be capable of providing potentially useful diagnostic information in patients with epilepsy.

Entropy is the measure of the degree of irregularity and disorder of analyzed time series. It is a nonlinear processing tool realized by evaluating the probability of finding $m$-similar patterns within the signal. Various Entropy descriptors were derived from the latter concept and used to process biomedical signals [7]. Recent studies have utilized entropy methods to detect epilepsy [1]. These methods included the Approximate Entropy (ApEn), Sample Entropy (SampEn) as being the standard entropy tools, among others [1]. Mekyska et al. have recently introduced kernel-based entropy measures and applied it to speech signals analysis [8]. Their work demonstrated that such entropy methods were sensitive to different signal phases or types.

To improve the classification of EEG signals and to predict seizure, it is essential to choose the best classifier [7]. How- 


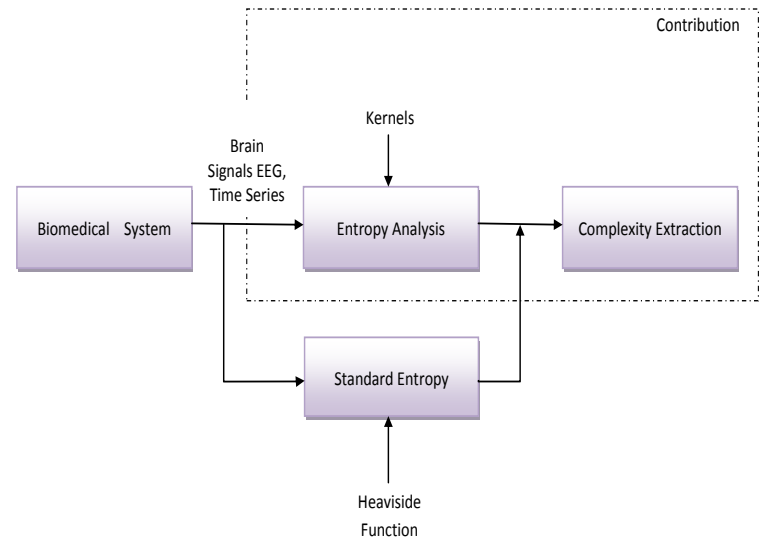

Fig. 1: The Block diagram of EEG complexity analysis through standard and kernel-based entropy.

ever, as classifiers require feature selection, and since there is no consensus on the choice of the features which lead to the most accurate results, it was essential for us to investigate the performance of existing nonlinear entropy features on our signals. By analogy to the work of Mekyska et al., this study aims to utilize some kernel-based entropy measures for feature extraction in sample EEG data.

We herein compare the standard entropy measures to kernelbased entropy methods on three distinct EEG phases corresponding to different physiological states: an pre-ictal state, an induced seizure state, and a post-ictal (after seizure) state. Four entropy features namely Approximate Entropy (ApEn), Sample Entropy (SampEn) and Renyi entropy of order 2 and 3, were implemented as the standard techniques. Three kernelbased features namely Triangular Entropy (TrEn), Spherical Entropy (SpEn) and Cauchy entropy (CaEn) were implemented to be tested on EEG recordings obtained from the three aforementioned states.

\section{Materials And Methods}

The major steps of this study are summarized in the block diagram in Fig. 1. Fig. 1 showcases the biomedical system, which is the brain, and the entropy analysis methods used to extract complexity parameters. Fig. 1 is divided into two parts, part one comprises the standard estimation of the entropy parameters such as ApEn, SampEn and others. The second part comprises the kernel-based entropy methods [8].

\section{A. Samples}

Three EEG sets were used from the recorded database of the American University of Beirut Medical Center (AUBMC). Three distinct phases were distinguished as the pre-ictal phase, the seizure (ictal) phase and post-ictal phase. The EEG signals were recorded during Electro-Convulsive Therapy (ECT), and a sample of the recordings is illustrated in Fig. 2. The various phases are characterized by the magnitude, temporal profile and the frequency of the dominant oscillations. In particular, EEG obtained during pre-ictal state is known to have large amplitude waves of low frequency $(1-3 \mathrm{~Hz}$, or delta range). Seizure-related EEG, on the other hand, shows stereotypical patterns that contain spikes of excessively large amplitude
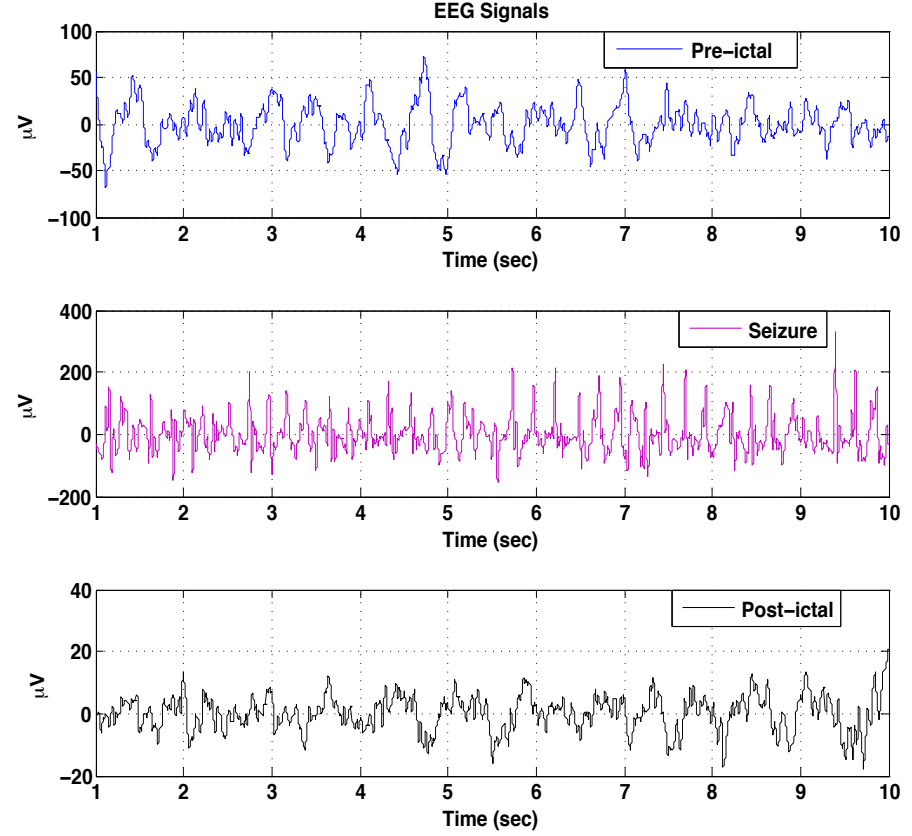

Fig. 2: EEG recordings. (Top) EEG during the pre-ictal phase, (Middle) EEG during the seizure phase and (Bottom) EEG during the post-ictal phase.

( $200 \mu \mathrm{V}$ ) followed by wave component and repeat at slightly higher frequencies $(2-5 \mathrm{~Hz})$. Finally, post-ictal EEG signals have more "quiet" random character with significantly reduced amplitudes and multitude of frequencies common in normal EEG recordings.

In each phase, 10 second recordings (sampled at a $1 \mathrm{KHz}$ rate) were utilized and windowed to produce sub-signals (or frames). For each signal, 1000 samples per second were produced and thus for 10 seconds 10000 samples were obtained. As the window size is 240 and the shift is $l=20$, 500 subsignals $(10000 / 20=500)$, each comprised 240 samples, were produced from each EEG. Provided that AUBMC data comprised 4 pre-ictal, 4 ictal and 4 post-ictal EEG recordings, 2000 pre-ictal, 2000 ictal and 2000 post-ictal EEG subsignals were used in the entropy analysis.

\section{B. Protocol}

The EEG recordings were obtained from subjects undergoing a clinical procedure known as ECT. This technique is highly effective treatment for patients with drug-resistant depression. During ECT, subjects are first anesthetized (first EEG phase), subsequently, brief repetitive large amplitude currents are administered through two stimulating electrodes located on scalp over the front-central brain areas. This electrical stimulation induces an epileptic seizure in the underlying cortical areas (second EEG phase) that then propagates into deep brain structures and lasts for a few seconds. Finally, the induced seizure is suppressed spontaneously by internal brain mechanisms leading to the post-ictal period (third EEG phase) during which the brain recovers its normal operation, albeit with reduced depression symptoms. 


\section{Entropy Complexity Analysis}

Each biomedical time series has its own information interpreted by the data in it or even by the regularity and the complexity of the signal. Entropy measures help in analyzing these signals by providing the amount of information they carry (Renyi Entropy) or by estimating the system complexity upon computing them (ApEn and SampEn and others).

1) Renyi Entropy: a mathematical generalization of Shannon Entropy which preserves the additivity of statistically independent systems and were compatible with Kolmogorov's probability axioms [8]. The Renyi entropy of order $\alpha$, where $\alpha \geq 0$ and $\alpha \neq 1$ is defined as:

$$
H_{\alpha}=\frac{1}{1-\alpha} \log \left(\sum_{i=(0)}^{n} P_{i}^{\alpha}\right)
$$

where $X$ is a discrete random variable with outcomes $1,2, \ldots \ldots, n$ and corresponding probabilities $P_{i}=\operatorname{Pr}(X=i)$ and $\log$ is based 2. Renyi Entropy has unique properties depending on the data spread and how the probability changes as order increases. Therefore, it is beneficial to study Renyi Entropy of order 2, 3 etc.

2) Approximate Entropy: is a method extensively used to assess the predictability and regularity of changes in a time series [8]. For a given time series of data $u(1), u(2), u(3), \ldots ., u(N)$, we define embedding dimension as the length of compared runs and denote it as $m$, and a filtering variable, $r$, which acts as the embedding tolerance. Furthermore a sequence of vectors, $X(i)$ is defined as:

$$
\mathbf{X}_{i}=[u(i), u(i+1), \ldots, u(i+m-1)],
$$

Furthermore $C_{i}^{m}$ is defined as:

$$
C_{i}^{m}=\frac{1}{N-m+1} \sum_{j=0}^{N-m+1} k_{(i, j, r)},
$$

for each $i, 1 \leq i \leq N-m+1$, we define the center of pressure position angle, $\phi^{m}(\mathrm{r})$, as:

$$
\phi^{m}(r)=\frac{1}{N-m+1} \sum_{i=1}^{N-m+1} \ln C_{i}^{m}(r)
$$

and then, ApEn is given as:

$$
A p \operatorname{En}(m . r)=\left[\phi^{m}(r)-\phi^{m+1}(r)\right] .
$$

It remains to define $\mathrm{k}(\mathrm{i}, \mathrm{j}, \mathrm{r})$ which is the kernel that's equal usually to the Heaviside function but can be changed to one of these kernels [9]:

Triangular Kernel:

$$
\mathbf{k}(i, j, r)=\left\{\begin{array}{cc}
1-\frac{\left.\| \mathbf{X}_{i}-\mathbf{X}_{j}\right) \|}{r} ; & \left.\| \mathbf{X}_{i}-\mathbf{X}_{j}\right) \| \leq r, \\
0, & \left.\| \mathbf{X}_{i}-\mathbf{X}_{j}\right) \|>r
\end{array}\right.
$$

the corresponding entropy is called Triangular Entropy and denoted through this work by (TrEn).

Spherical Kernel:

$$
\mathbf{k}(i, j, r)=\left\{\begin{array}{c}
1-\frac{3}{2} \frac{\left.\| \mathbf{X}_{i}-\mathbf{X}_{j}\right) \|}{r}+\frac{1}{2}\left(\frac{\left.\| \mathbf{X}_{i}-\mathbf{X}_{j}\right) \|}{r}\right)^{3} \\
\left.\| \mathbf{X}_{i}-\mathbf{X}_{j}\right) \| \leq r \\
\left.0, \quad \quad \| \mathbf{X}_{i}-\mathbf{X}_{j}\right) \|>r
\end{array}\right.
$$

the corresponding entropy is called Spherical Entropy and denoted by (SpEn).

Cauchy Kernel:

$$
\mathbf{k}(i, j, r)=\left\{\begin{array}{c}
\frac{1}{1+\frac{\left.\left(\| \mathbf{X}_{i}-\mathbf{X}_{j}\right) \|\right)^{2}}{r}} ;\left\|\mathbf{X}_{i}-\mathbf{X}_{j}\right\| \leq r, \\
\left.0, \quad \| \mathbf{X}_{i}-\mathbf{X}_{j}\right) \|>r
\end{array}\right.
$$

the corresponding entropy is called Cauchy Entropy and denoted by (CaEn).

3) Sample Entropy: due to the self comparison of a time series template vector with itself, ApEn values are interpreted to be more regular than they actually are. This disadvantage of ApEn was avoided using Sample Entropy (SampEn), SampEn measures the complexity without including self similar patterns.

$$
\operatorname{SampEn}(m . r)=\left[\phi^{m}(r)-\phi^{m+1}(r)\right],
$$

where $\phi^{m}(r)$ is given by Eq. 4 yet:

$$
C_{i}^{m}=\frac{1}{N-m+1} \sum_{j=0, i \neq j}^{N-m+1} k_{(i, j, r)},
$$

The algorithm builds up runs of points matching within the tolerance $r$ until there is not a match, and keeps track of template matches continued until the end of the data [7].

\section{RESULTS}

To increase the sensitivity of this comparative study, the entropy analysis has been applied on each sub-signal (frame) produced after windowing the EEG recordings.

\section{A. Quantification Comparison}

Table I reports the seven average entropy values of the EEG during the pre-ictal (pre-seizure), the Seizure and the post-ictal (post-seizure) phases of the recordings using the standard and kernel based methods. For each En method used, averaged entropy values are reported for the three phases. For ApEn, the asterisk is associated with the distinctly higher value and corresponds to the Normal EEG (0.309). Similarly, for SampEn, SpEm, and CaEn the asterisk is also associated with the value obtained from the Normal EEG $(0.075,20.00$ and 0.025 , respectively).

\section{B. Relative Difference Comparison}

The mean measure of irregularity corresponding to the average value obtained for all sub-signals (frames) extracted from the 10 second original data (in each of pre-ictal, seizure and post-ictal) has been used to compare the relative difference between phases. The relative difference $R D(E n)$ (in \%) is defined as follows:

$$
R D(E n)=\frac{\left|\bar{E} n\left(E E G_{\text {phase } i}\right)-\bar{E} n\left(E E G_{\text {phase } j}\right)\right|}{\bar{E} n\left(E E G_{\text {phase } j}\right)},
$$

where $\mathrm{i}=\{$ Anaesthesia, Seizure, Normal $\}$ and $i \neq j$.

Table II summarizes the relative difference of the seven previous parameters. The important RD values were highlighted in bold. $\mathrm{RD}(\mathrm{En})$ 's are arranged in an ascending order of seizure - post-ictal phase separation: $R D(\operatorname{TrEn})<R D(A p E n)<$ $R D(C a E n)<R D($ RenyiEn-order 2$)<R D($ RenyiEn- 
TABLE I: The average entropy values of the EEG during pre-ictal, seizure and post-ictal phases using the standard and kernel based methods.

\begin{tabular}{|c|c|c|c|}
\hline Average Entropy & Pre-Ictal EEG & Seizure EEG & Post-Ictal EEG \\
\hline \hline ApEn & 0.045 & 0.163 & $0.309^{*}$ \\
\hline SampEn & 0.004 & 0.004 & $0.075^{*}$ \\
\hline Renyi-En (order 2) & 11.25 & 13.50 & 8.647 \\
\hline Renyi-En (order 3) & 7.020 & 8.539 & 5.084 \\
\hline TrEn & 0.691 & 0.691 & 0.690 \\
\hline SpEn & 0.010 & 0.040 & $20.00^{*}$ \\
\hline CaEn & 0.016 & 0.010 & $0.025^{*}$ \\
\hline
\end{tabular}

TABLE II: The relative difference (RD) and normalized values (in \%) of the average entropy between EEG during pre-ictal and seizure, EEG during seizure and post-ictal phases and EEG during pre-ictal and post-ictal phases.

\begin{tabular}{|l|l|l|l|l|l|l|}
\hline Relative Difference & $\begin{array}{l}\text { Pre-Ictal } \\
-\quad \text { Seizure } \\
\text { EEG }\end{array}$ & $\begin{array}{l}\text { Seizure } \\
\text { Post-Ictal } \\
\text { EEG }\end{array}$ & $\begin{array}{l}\text { Pre-Ictal - } \\
\text { Post-Ictal } \\
\text { EEG }\end{array}$ & $\begin{array}{l}\text { Normalized } \\
\text { Values } \\
\text { Pre-Ictal - } \\
\text { Seizure }\end{array}$ & $\begin{array}{l}\text { Normalized } \\
\text { Values } \\
\text { Seizure } \\
\text { Post-Ictal }\end{array}$ & $\begin{array}{l}\text { Normalized } \\
\text { Values } \\
\text { Pre-Ictal } \\
\text { Post-Ictal }\end{array}$ \\
\hline \hline RD(ApEn) & $\mathbf{2 . 6 1 0}$ & 0.4725 & 0.8544 & $\mathbf{8 7 . 0 0 \%}$ & $47.27 \%$ & $\mathbf{8 5 . 4 6 \%}$ \\
\hline RD(SampEn) & 0.000 & $\mathbf{0 . 9 4 6 7}$ & $\mathbf{0 . 9 4 6 7}$ & $0.00 \%$ & $\mathbf{9 4 . 8 5 \%}$ & $\mathbf{9 4 . 7 1 \%}$ \\
\hline RD(Renyi-En (order 2)) & 0.200 & 0.5612 & 0.3010 & $6.67 \%$ & $56.17 \%$ & $30.02 \%$ \\
\hline RD(Renyi-En (order 3)) & 0.216 & 0.6796 & 0.3808 & $7.20 \%$ & $68.05 \%$ & $38.01 \%$ \\
\hline RD(TrEn) & 0.000 & 0.0014 & 0.0014 & $0.00 \%$ & $0.00 \%$ & $0.00 \%$ \\
\hline RD(SpEn) & $\mathbf{3 . 0 0 0}$ & $\mathbf{0 . 9 9 8 0}$ & $\mathbf{0 . 9 9 9 5}$ & $\mathbf{1 0 0 . 0 0 \%}$ & $\mathbf{1 0 0 . 0 0 \%}$ & $\mathbf{1 0 0 . 0 0 \%}$ \\
\hline RD(CaEn) & 0.3750 & 0.6000 & 0.3600 & $12.50 \%$ & $60.06 \%$ & $35.93 \%$ \\
\hline
\end{tabular}

order 3) $<R D(S a m p E n)<R D(S p E n)$. Moreover, $\mathrm{RD}(\mathrm{En})$ 's are arranged in an ascending order of pre-ictal seizure phase separation: $R D(S a m p E n)=R D(\operatorname{TrEn})<$ $R D($ RenyiEn - order 2) $<R D($ RenyiEn - order 3$)<$ $R D(C a E n)<R D(A p E n)<R D(S p E n)$.

\section{DISCUSSION AND CONCLUSION}

The findings derived from Table II suggest that the best parameter that emphasizes the distinction between normal and seizure EEG data was $R D(S p E n)$. Therefore, it was advantageous to choose a spherical kernal-based entropy over the standard ApEn and the other presented alternatives. Note that $R D(S a m p E n)$ surpasses ApEn, while in pre-ictal seizure phases ApEn surpasses SampEn. Therefore, the choice of the feature is dependent on the application at hand.

SpEn kernel-based entropy feature showed the highest sensitivity to EEG (Seizure-Normal) phase changes with the highest normalized relative separation (100\%). Among the alternative entropy measures studied, the sample entropy feature (SamEn) exhibited the most sensitive to EEG phase changes with the highest relative separation $(94.85 \%)$.

The large scores (asterisk) of the four entropy measures Table I were in accordance with the medical evidence that Normal EEG signals are more irregular and complex than EEG in a seizure phase.

Finally, we note that yet other entropy features can be studied and compared with the most sensitive parameters provided in this work. Still, the representation power of the extracted features (measures), as part of an automated analysis procedure, needs to be tested in a variety of classifiers, such as Support Vector Machine and K-Nearest Neighbour. Moreover, additional subjects should be considered in the analysis.

\section{REFERENCES}

[1] U. R. Acharya, F. Molinari, S. V. Sree, S. Chattopadhyay, K.-H. Ng, and J. S. Suri, "Automated diagnosis of epileptic eeg using entropies," Biomedical Signal Processing and Control, vol. 7, no. 4, pp. 401-408, 2012.

[2] J. C. Garcia-Monco, I. E. Cortina, E. Ferreira, A. Martínez, L. Ruiz, A. Cabrera, and M. G. Beldarrain, "Reversible splenial lesion syndrome (resles): what's in a name?" Journal of Neuroimaging, vol. 21, no. 2, pp. e1-e14, 2011.

[3] D. Coyle, T. M. McGinnity, and G. Prasad, "Improving the separability of multiple eeg features for a bci by neural-time-series-predictionpreprocessing," Biomedical Signal Processing and Control, vol. 5, no. 3 , pp. 196-204, 2010.

[4] A. Babloyantz, "Evidence of chaotic dynamics of brain activity during the sleep cycle," in Dimensions and entropies in chaotic systems. Springer, 1986, pp. 241-245.

[5] D. Subha, K. Joseph, U. Acharya, and C. Lim, "Eeg signal processing: A survey," Journal of Medical Systems, vol. 34, no. 2, pp. 195-212, 2010.

[6] R. Andrzejak, G. Widman, K. Lehnertz, C. Rieke, P. David, and C. Elger "The epileptic process as nonlinear deterministic dynamics in a stochastic environment: an evaluation on mesial temporal lobe epilepsy," Epilepsy Research, vol. 44, no. 2, pp. 129-140, 2001.

[7] L. Chen, Y. Zhao, J. Zhang, and J.-Z. Zou, "Automatic detection of alertness/drowsiness from physiological signals using wavelet-based nonlinear features and machine learning," Expert Systems with Applications (2015), doi: http://dx.doi.org/10.1016/j.eswa.2015.05.028.

[8] J. Mekyska, E. Janousova, P. Gomez-Vilda, Z. Smekal, I. Rektorova, I. Eliasova, M. Kostalova, M. Mrackova, J. B. Alonso-Hernandez, M. Faundez-Zanuy, and k. Lopez-de Ipia, "Robust and complex approach of pathological speech signal analysis," Neurocomputing, (2015), doi:10.1016/j.neucom.2015.02.085.

[9] K. Adjerid, P. Fino, M. Habib, A. Rezaei, S. Ross, and T. Lockhart, "Comparing postural stability entropy analyses to differentiate fallers and non-fallers," 2014. 